the cell increased rapidly with the wave-length. The maximum reverse current obtained from the marginal regions of the cell was no less than 28.5 per cent of the maximum direct current obtained for the same regions.

We should like to take this opportunity of apologising to Dr. P. Auger and M. C. Lapicque for having overlooked the section of their paper ${ }^{1}$ in which they briefly mention the reversal phenomenon, and give its explanation, identical with the one since given by us and by Mr. Guild. Dr. Auger also studied the spectral transparency of such gold and silver films, as Mr. Guild is now doing; from this he deduced the true variation in the spectrum of the photo-electric properties of cuprous oxide, since the curves given by gold and silver film cells were identical when corrected for the transmission of the film. The transmission of euprous oxide films has been studied by L. Dubar ${ }^{2}$, who investigated also the temperature coefficient of the cells up to $60^{\circ} \mathrm{C}$. Dubar ${ }^{3}$ has also studied the peculiar constitution of the cuprous oxide layer.

Royal Dublin Society.

H. H. PoOLE.

Marine Biological Laboratory,

Plymouth.

March 11.

'C. R. Acad. Sci. Paris, 193, 320; 1931.
2 Loc. cit., 193, 659; 1931.
3 Loc, cit., 194, $1332 ; 1932$.

\section{A Rule for the Rotatory Direction of the Acetylated} Aldonic Nitriles

DURING research on some sugar derivatives, the rotation in chloroform of their acetylated aldonic nitriles was determined, and the following results were obtained :

tetra-acetyl $d$-xylonic nitrile, tetra-acetyl $l$-arabonic nitrile, penta-acetyl $d$-galactonic nitrile,

$(\alpha)_{D}^{23}=+50 \cdot 3^{\circ}$;

$(\alpha)_{D}^{22}=+4 \cdot 2^{\circ}$;

$(\alpha)_{D}^{22}=+48 \cdot 4^{\circ}$.

From a search in the literature, the rotations determined for other nitriles were found to be:

penta-acetyl $d$-gluconic nitrile ${ }^{1,},{ }^{2},(\alpha)_{D}^{22}=+46 \cdot 2^{\circ}:+48 \cdot 0^{\circ}$; hexa-acetyl $d$-gluco- $\alpha$-heptonic nitrile ${ }^{4},(\alpha)_{D}^{21}=+24 \cdot 6^{\circ}$; hexa-acetyl $d$-manno- $\alpha$-heptonicnitrile ${ }^{3}(\alpha)_{D}^{20}=+31 \cdot 4^{\circ}$; penta-acetyl $d$-mannonic nitrile ${ }^{2}, \quad(\alpha)_{D}^{30}=-\mathbf{I} \cdot 4^{\circ}$; penta-acetyl $l$-rhamno- $\alpha$-hexonicnitrile ${ }^{3},(\alpha)_{D}^{30}=-76 \cdot 4^{\circ}$.

The following data were found for non-acetylated aldonic nitriles:

gluconic nitrile*

$d$-manno- $\alpha$-heptonic nitrile ${ }^{3}$,

$(\alpha)_{D}^{21}=+8 \cdot 8^{\circ}$

$(\alpha)_{D}^{20}=+23 \cdot 1^{\circ}$;

$(\alpha)_{D}^{20}=-23 \cdot 4^{\circ}$.

$l$-rhamno- $\alpha$-hexonic nitrile ${ }^{3}$,

When the figures and signs of rotation of the acetylated nitriles are compared, it is seen that a new rule similar to the well-known amide or phenylhydrazide rules of Hudson for aldonic acids, can also be formulated for the acetylated nitriles of the same acids.

When the configuration of the 2-carbon atom is considered, it follows that nitriles in which that atom has the configuration $\mathrm{HCO} . \mathrm{COCH}_{3}$ (that is, $\mathrm{HCOH}$ in the primitive monose, employing Fischer's projection formula) rotates to the right, as is found in $d$-xylonic, $l$-arabonic, $d$-galactonic, $d$-gluconic, $d$-gluco$\alpha$-heptonic, and $d$-manno- $\alpha$-heptonic acetylatednitriles.
When the configuration of the 2-carbon atom is reversed: $\mathrm{CH}_{3} \mathrm{CO} . \mathrm{OCH}$ (that is, HOCH in the primitive monose) the nitriles rotate to the left, as in $d$-mannonic, and $l$-rhamno- $\alpha$-hexonic acetylated nitriles. It follows that the sign of rotation of the nitriles will be the same as that of the amides and phenylhydrazines of the same acids. It is of no use, however, to make comparisons between the rotatory values of the acetylated nitriles and the amides or the free acids, owing to the different solvents employed in each case.

The theoretical foundations of the nitrile rule are the same as those for the amides or the phenylhydrazides, and have been explained by Kuhn and Freudenberg. Only exaltation of the individual asym. metric carbon atoms must happen owing to acetylation of the alcoholic hydroxyls. When the free nitriles are considered, it is seen that perhaps the rule can also be applied to them, although the examples are few in number.

Work is now being done with other members of the same group to see if they accord, as we expect, to the rule. It is hoped to include $\operatorname{som} \theta \alpha$-oxy acids.

Instituto de Fisiología, Venancio Deulofeu. Buenos Aires.

$$
\text { Jan. } 16 .
$$

I Zemplen and Kiss, Ber., 60, $165 ; 1927$

${ }_{2}$ Wolfrom and Thompson, J. Amer. Chem. Soc., 53, 622; 1931

${ }^{3}$ Mikšic, Chem. Zentr., 1, 2704; 1928. Chem. Ab8., 23, 2941; 1929

- Zemplen, Ber. 60, 171 ; 1927 .

\section{Zones of Apparent Inhibition of Sunspots on the Solar Disc}

IN a recent paper ${ }^{1}$ referred to in NATURE of February 18, p. 245, Mr. P. R. Chidambara Aiyar reports the result of an analysis of the distribution over the visible portion of the sun of sunspots lasting two days, and finds that his curves of distribution show, in addition to a maximum at the centre of the disc, maxima at longitudes about $15^{\circ}$ inside either limb. This he describes in terms of the intervening minima by calling their positions zones of apparent inhibition of spots.

It is to be expected that the number of spots of any given size or period observed, in an assigned number of degrees of longitude, would show a steady diminution from centre to limb simply on account of foreshortening rendering the spots less visible. The minima noted by Mr. Aiyar can scarcely have a physical significance since the co-ordinates used (apparent longitude on the solar disc) have no physical meaning for the sun, being fixed on any day by the position of the earth in its orbit on that day.

Mr. Aiyar has established that his results are not due to random variations in the data, and accordingly a cause lying in the mode of selection of the data is to be sought. I wish to point out that the explanation of the inhibitory zones is to be found in the following reason for the existence of the maxima near the limb, and that there is in fact no region of scarcity, but merely a spurious region of richness.

In selecting the spots, only those are used which are observed on two days, but not on three or more. If the whole surface of the sun were visible this selection would operate uniformly in all solar longitudes, but in fact only the region from $-90^{\circ}$ to $+90^{\circ}$ is visible at any one time, and this leads to an excessive count near the limb. Owing to the rotation 